\title{
Hydrodynamic theory for granular gases *
}

\author{
Rosa Ramírez ${ }^{1,2}$, Dino Risso ${ }^{3}$, Rodrigo Soto ${ }^{2}$ and Patricio Cordero ${ }^{1}$ \\ ${ }^{1}$ Departamento de Física, Universidad de Chile, Santiago, Chile \\ ${ }^{2}$ CECAM, ENS-Lyon, 46 Allée d'Italie, 69007, France \\ ${ }^{3}$ Departamento de Física, Universidad del Bío-Bío, Concepción, Chile
}

\begin{abstract}
A granular gas subjected to a permanent injection of energy is described by means of hydrodynamic equations derived from a moment expansion method. The method uses as reference function not a Maxwellian distribution $f_{\mathrm{M}}$ but a distribution $f_{0}=\Phi f_{\mathrm{M}}$, such that $\Phi$ adds a fourth cumulant $\kappa$ to the velocity distribution. The formalism is applied to a stationary conductive case showing that the theory fits extraordinarily well the results coming from our molecular dynamic simulations once we determine $\kappa$ as a function of the inelasticity of the particle-particle collisions. The shape of $\kappa$ is independent of the size $N$ of the system.
\end{abstract}

\section{INTRODUCTION}

Granular systems subjected to a sufficiently strong excitation may have a fluid-like behavior [1,22]. From the very beginning several authors have attempted to derive hydrodynamic equations for these systems [3,4]. If the excitation of the granular system is through a permanent injection of energy, the fluid system may stabilize to a low density stationary gaseous state which necessarily is a nonequilibrium state and it usually is inhomogeneous as well. To develop the basic features of the theory of gaseous granular systems we restrict the analysis to the simplifying inelastic hard sphere model (IHS) [3].

Many authors studying granular gases have put particular attention to studying the spontaneous homogeneous cooling of a granular system using periodic boundary conditions [5.,6]. This time dependent state is called homogeneous cooling state (HCS) and the understanding of its properties has been improving through many articles [7] [10]. A crucial breakthrough was the realization by Goldshtein and Shapiro [7] that the homogeneous cooling distribution function has a scaling property with respect to the instantaneous temperature. Such distribution - which we will be calling $f_{\mathrm{HCS}}$ - is known in approximate forms [11.12]. It is known, among other things, that its fourth cumulant $\kappa$ does not vanish and that it has a long velocity tail.

A nonequilibrium inhomogeneous gaseous system, on the other hand, is described by a distorted distribution function typically obtained from Boltzmann's equation expanding the distribution either in gradients of the hydrodynamic fields (Chapmann-Enskog's method) [13] or making a moment expansion (Grad's method) [14]. For normal gases the expansion is made about the equilibrium Maxwell's distribution.

In this article we will assume that a low density nonequilibrium granular system has a local distribution function which can be obtained expanding about a distribution $f_{0}$ resembling a $f_{\mathrm{HCS}}$ in the sense that it has a significantly nonvanishing fourth cumulant. We introduce a reference function (see Eq. (5) below) which is a Maxwellian distorted by a factor which incorporates the next non trivial cumulant, a fourth cumulant, to the distribution function and then we undertake a perturbative moment expansion à la Grad about $f_{0}$ to solve Boltzmann's equation. Some authors have done some calculations in this direction but using the gradient expansion (Chapman-Enskog) method [15]. The point is that, without the notion of equilibrium, we expect that the reference state, $f_{0}$ in our case, should resemble more the homogeneous cooling state than the simple Maxwellian.

We study a two-dimensional system of hard disks, and the moment expansion - in dimension two - is an 8 moment expansion: the number density $n(\vec{r}, t)$, the velocity field $\vec{v}(\vec{r}, t)$, the granular temperature field $T(\vec{r}, t)$, the pressure tensor $P_{i j}(\vec{r}, t)$ and the heat flux vector field $\vec{Q}(\vec{r}, t)$. The dynamic variables are not the components of the pressure tensor $\mathbb{P}$ itself but the components of the symmetric traceless part $p_{i j}$ where $P_{i j}=p \delta_{i j}+p_{i j}$ and $p$ is the hydrostatic pressure.

*URL: http://www.cec.uchile.cl/cinetica/ 
As it can be seen in Grad's article [14] or in [16] the method yields hydrodynamic equations for all the fields mentioned above. In particular, the dynamic equations for $p_{i j}$ and $\vec{Q}$ take the place of what would normally be the constitutive (transport) equations of standard hydrodynamics. This last point means that we are not assuming any constitutive equations whatsoever, their present counterparts are dynamic equations.

It is well established that, in the case of the IHS model for granular systems, Boltzmann's equation is modified in that the restitution coefficient $r$ enters solely in the gain term of the collision integral and it does so in two forms. First the gain term has an overall factor $r^{-2}$ and second, the distribution functions appearing in the gain term depend upon the precollision velocities, and these velocities depend on $r$ 何.

When the modified Boltzmann's equation is used the stemming hydrodynamic equations get factors that depend on the inelasticity coefficient $q$,

$$
q=\frac{1-r}{2}
$$

( $q=0$ in the perfectly elastic case) except that the mass continuity equation and the momentum balance equation remain unchanged since mass and momentum continue being microscopically conserved.

In the context of Boltzmann's equation a dissipative gas satisfies the ideal gas equation of state

$$
p=n T
$$

where the granular temperature $T$ is defined in energy units as the average kinetic energy per particle. If we were to consider the Boltzmann-Enskog equation, then the inelasticity coefficient would enter through the Enskog collision factor $\chi$, and the equation of state of a normal gas and a dissipative gas would differ, but in the present context Eq. (2) holds.

Usual moment expansion methods (as Grad's is) are appropriate to describe bulk properties. Wall effects are not well described unless higher order momenta are included in the expansion, which are not trivial to handle [17]. Already in normal gases hydrodynamic fields may have discontinuities at walls. In a previous article we gave a kinetic description of a one-dimensional granular system with theoretical tools such that our description was correct and precise up to the walls 18] but we have not generalized yet that type of formalism to higher dimensions, hence, in the present article, we use moment expansions.

In consequence the formalism we are going to present suffers too of the weakness of moment expansions: it is unreliable precisely at the points where the boundary conditions should be imposed forcing us to trade the boundary conditions for conditions imposed far from the walls based on the actual behavior of the system according to our molecular dynamic simulations.

Our moment expansion method, explained in detail in Sec. II, uses as reference distribution function a distribution $f_{0}$ which differs from a Maxwellian distribution in that it has a nonvanishing fourth cumulant, $\kappa$.

The method leads to hydrodynamic equations for low density granular systems that depend parametrically on the inelasticity coefficient $q$ and $\kappa$. We apply this hydrodynamic equations to a stationary and purely conductive case as in [19]. The value of the fourth cumulant $\kappa$, or better, the dependence of the fourth cumulant on $q$ is determined directly from molecular dynamic simulations, and it turns out to be independent of the size $N$ of the system. The predictions that follow from our formalism agree very well with all our simulational data.

In Sec. II we briefly present the moment expansion method, in Sec. III the hydrodynamic equations that follow are given and specialized to a purely conductive case and finally in Sec. IV theory and simulational results are compared. Final comments are in Sec. V.

\section{THE MOMENT EXPANSION METHOD FOR GRANULAR SYSTEMS}

Moment expansion methods can summarily be described as follows. Take a velocity distribution function $f_{0}(\vec{r}, \vec{c}, t)$ which is considered to be the reference function about which an expansion is going to be made. For normal gases the natural choice for $f_{0}$ is a local Maxwellian distribution $f_{\mathrm{M}}$ written in terms of the peculiar velocity $\vec{C}=\vec{c}-\vec{v}(\vec{r}, t)$, where $\vec{v}(\vec{r}, t)$ is the hydrodynamic velocity. Next a set of orthonormal polynomials on $\vec{C}, H_{a}(\vec{C})$, are built in the sense that $H_{0}=1$ and

$$
\int H_{a}(\vec{C}) H_{b}(\vec{C}) f_{0}(\vec{r}, \vec{C}, t) d \vec{C}=\delta_{a b}
$$

The polynomials $H_{a}$ are obtained simply building a base of orthonormal polynomials starting from $H_{0}=1$ and from first degree upwards. When $f_{0}$ is a Maxwellian the $H_{a}$ are Hermite polynomials but in general they are not. 
Then a solution of the form

$$
f(\vec{r}, \vec{C}, t)=\left(1+\sum_{a} H_{a}(\vec{C}) R_{a}(\vec{r}, t)\right) f_{0}(\vec{r}, \vec{C}, t)
$$

is replaced in Boltzmann's equation. The $R_{a}$ are the moments of $f$ with respect to the $H_{a}$ and they can directly be related to the moments associated to $\vec{c}$ (the velocity field $\vec{v}$ ), to $\rho C_{i} C_{j}$ (the kinematic pressure tensor $P_{i j}$ ) and to $\frac{1}{2} \rho C^{2} \vec{C}$ (the heat flux vector $\vec{Q}$ ). One could go on but we have used polynomials only containing $C_{i}, C_{i} C_{j}$ and $C^{2} C_{i}$ as Grad did.

The following step is to derive integrability conditions multiplying the kinetic equation consecutively by the $H_{n}$ and then integrating the equation over $\vec{C}$. The idea is to do this up to a given order and drop all contributions coming from polynomials of degree higher than a chosen value (up to order 3 in our case). This gives a set of hydrodynamic equations for the different moments.

A key point is the choice of the reference function $f_{0}$. The two dimensional Maxwellian $f_{\mathrm{M}}=$ $n \frac{m}{2 \pi T} \exp \left[-m C^{2} /(2 T)\right]$ is privileged as the solution describing the equilibrium state of a normal gas. Since in granular systems there is no such thing as equilibrium a next best choice, seems a distorted Maxwellian distribution [11]

$$
f_{0}=\left[1+\frac{\kappa}{2}\left(1-\mathcal{C}^{2}+\frac{\mathcal{C}^{4}}{8}\right)\right] f_{\mathrm{M}}
$$

where the dimensionless peculiar velocity is

$$
\overrightarrow{\mathcal{C}}=\sqrt{\frac{m}{T}} \vec{C}
$$

and $T$ is the granular temperature. The coefficient $\kappa$ is the fourth cumulant of $f_{0}$ and it depends on the inelasticity coefficient $q$ while the coefficients in front of $\mathcal{C}^{2}$ and $\mathcal{C}^{4}$ are derived from requiring that $f_{0}$ is normalized and that $\left\langle\frac{m}{2} C^{2}\right\rangle_{f_{0}}=T$. The fourth cumulant $\kappa$ in dimension two is

$$
\kappa=\frac{\left\langle\mathcal{C}^{4}\right\rangle-2\left\langle\mathcal{C}^{2}\right\rangle^{2}}{\left\langle\mathcal{C}^{2}\right\rangle^{2}},
$$

In the case of the homogeneous cooling state, recent articles have justified explicit forms 11. 12] for $\kappa$ in the context of distribution functions like $f_{0}$. Their results are well approximated by

$$
\kappa=\frac{b_{1}+b_{2} q}{1+b_{3} q} q
$$

with

$$
b_{1}=-2, \quad b_{2} \approx 13.619, \quad b_{3} \approx 4.5969 .
$$

The rational expression given in (8) is valid within $2.9 \%$ for $q \leq 0.08, r=1-2 q=0.84$. Notice that $\kappa(q=0)=0$ allowing to recover the elastic case.

We describe quite satisfactorily nonhomogeneous, nonequilibrium stationary states with a $\kappa$ as in (8), but with numerical factors different from those in Eq. (91). The latter values are valid only for the homogeneous cooling state while our system is kept in a stationary inhomogeneous state with appropriate boundary conditions.

For any $\kappa$ the resulting distribution obtained with the method summarized above is

$$
\begin{aligned}
f^{(\kappa)}= & {\left[1+\frac{p_{x x}}{p(2+\kappa)}\left(\mathcal{C}_{x}^{2}-\mathcal{C}_{y}^{2}\right)+2 \frac{p_{x y}}{p(2+\kappa)} \mathcal{C}_{x} \mathcal{C}_{y}\right.} \\
& \left.+\frac{Q_{x}}{2 Q_{0}} \frac{\left(\mathcal{C}^{2}-4-2 \kappa\right) \mathcal{C}_{x}}{\left(2+5 \kappa-\kappa^{2}\right)}+\frac{Q_{y}}{2 Q_{0}} \frac{\left(\mathcal{C}^{2}-4-2 \kappa\right) \mathcal{C}_{y}}{\left(2+5 \kappa-\kappa^{2}\right)}\right] f_{0}
\end{aligned}
$$

where, $p_{y y}=p_{x x}$ and $Q_{0}=\sqrt{\frac{T}{m}} p$. The distribution $f^{(\kappa)}$ shares with $f_{0}$ the first scalar moments: density, temperature and fourth cumulant, for any value of $q$. If $\kappa$ is chosen to be zero then, in Eq. (10), $f_{0} \rightarrow f_{\mathrm{M}}$ and $f^{(\kappa)}$ becomes the usual Grad's distribution. Hence the whole method would be the original method devised by Grad and, if $\kappa$ is chosen to be Eq. (8) with coefficient values as in Eq. (9), then $f_{0}$ would be what we are calling $f_{\mathrm{HCS}}$.

Given our ignorance regarding granular gases one could, in principle, accept $f_{\mathrm{M}}$ or $f_{\mathrm{HCS}}$ as legitimate reference functions to make the moment expansion. In the following sections we compare the three formalisms (reference functions $f_{\mathrm{M}}, f_{\mathrm{HCS}}$ and $f_{0}$, the latter with a $\kappa$ adjusted to the results) concluding that only the one based on $f_{0}$ gives acceptable results for a sufficiently large range of $q$. In fact they are very good. 


\section{THE HYDRODYNAMIC EQUATIONS}

As already mentioned, the inelasticity coefficient $q$ enters the kinetic equation in two different forms. It appears as a factor $\frac{1}{r^{2}}=\frac{1}{(1-2 q)^{2}}$ in the gain term and it appears in the expression for the precollision velocities which are part of the argument of the distribution functions appearing in the gain term. When the solution $f^{(\kappa)}$ is inserted in Boltzmann's equation, $q$ enters in a still third form, precisely through the $\kappa$ coefficient given by Eqs. (8) and (10). Expanding the collisonal term of Boltzmann's equation in powers of $q$ and $\kappa$, the moment method yields the following hydrodynamics equations for a granular gas

$$
\begin{gathered}
\frac{D n}{D t}+n \nabla \cdot \vec{v}=0 \\
m n \frac{D \vec{v}}{D t}-n \vec{F}+\nabla \cdot \mathbb{P}=0 \\
n \frac{D T}{D t}+\nabla \cdot \vec{Q}+\mathbb{P}: \nabla \vec{v}=-\frac{2 A(q) n T}{\tau}, \\
\frac{\partial p_{i j}}{\partial t}+\frac{\partial}{\partial x_{k}}\left(v_{k} p_{i j}\right)+\frac{1}{2}\left(\frac{\partial Q_{i}}{\partial x_{j}}+\frac{\partial Q_{j}}{\partial x_{i}}-\delta_{i j} \frac{\partial Q_{k}}{\partial x_{k}}\right)+ \\
p_{r j} \frac{\partial v_{i}}{\partial x_{r}}+p_{r i} \frac{\partial v_{j}}{\partial x_{r}}-\delta_{i j} p_{r s} \frac{\partial v_{s}}{\partial x_{r}}+p\left(\frac{\partial v_{i}}{\partial x_{j}}+\frac{\partial v_{j}}{\partial x_{i}}-\delta_{i j} \frac{\partial v_{r}}{\partial x_{r}}\right)=-\frac{B(q)}{\tau} p_{i j}, \\
\frac{\partial Q_{k}}{\partial t}+\frac{\partial}{\partial x_{r}}\left(v_{r} Q_{k}\right)+\frac{3}{2} \frac{\partial v_{k}}{\partial x_{r}} Q_{r}+\frac{1}{2} \frac{\partial v_{r}}{\partial x_{k}} Q_{r}+\frac{1}{2} \frac{\partial v_{r}}{\partial x_{r}} Q_{k}+ \\
\frac{T}{m} \frac{\partial p_{k r}}{\partial x_{r}}+\frac{3 p_{k r}}{m} \frac{\partial T}{\partial x_{r}}-\frac{p_{k r}}{m n} \frac{\partial P_{r s}}{\partial x_{s}}+\frac{3 p}{m} \frac{\partial T}{\partial x_{k}}=-\frac{C(q)}{2 \tau} Q_{k},
\end{gathered}
$$

where $D / D t \equiv \partial / \partial t+\vec{v} \cdot \nabla$

$$
\tau=\frac{1}{2 \sigma p} \sqrt{\frac{m T}{\pi}}
$$

is a characteristic relaxation time, $\sigma$ is the diameter of the particles, and the coefficients $A, B$ and $C$ for the generic distribution $f^{(\kappa)}$ are

$$
\begin{aligned}
& A(q)=\left[q(1-q)+\mathcal{O}\left(q^{5}\right)\right]\left(1+\frac{3}{32} \kappa+\frac{9}{4096} \kappa^{2}\right) \\
& B(q)=\frac{1+\frac{1}{2} q-\frac{3}{2} q^{2}+\frac{23}{32} \kappa\left(1+\frac{q}{2}\right)+\frac{\kappa^{2}}{4096}+\mathcal{O}\left(q^{3}\right)+\mathcal{O}\left(\kappa^{3}\right)}{\left(1+\frac{1}{2} \kappa\right)} \\
& C(q)=\frac{1+\frac{13}{2} q-\frac{15}{2} q^{2}+\frac{\kappa}{64}(206+1267 q)-\frac{2415}{4096} \kappa^{2}+\mathcal{O}\left(q^{3}\right)+\mathcal{O}\left(\kappa^{3}\right)}{\left(1+\frac{5}{2} \kappa-\frac{\kappa^{2}}{2}\right)} .
\end{aligned}
$$

They depend on $q$ and $\kappa$, but $\kappa$ depends on $q$. At least for small $q$ the coefficients $A, B$ and $C$ are positive $(q$ varies between 0 and $\frac{1}{2}$ ). The coefficient $A$ in Eq. (13) determines the energy dissipation in the system and consequently it vanishes in the elastic limit while $B$ and $C$ tend to 1 . In the previous equations both $p_{i j}$ and $P_{i j}$ appear depending on which of them gives a more compact expression.

It is our hope that the above hydrodynamics is valid in a wide variety of situations compatible with gaseous states and no clustering [5.8] but in this article we restrict our study to a hydrostatic case.

We are going to consider the purely conductive regime, with no external force, $\vec{F}=0$. The system of $N$ disks is in a rectangular box of dimension $L_{x} \times L_{y}$, with thermal walls, at $y=-\frac{1}{2} L_{y}$ and at $y=\frac{1}{2} L_{y}$, both at temperature $T_{0}$, and periodic boundary conditions in the $X$ direction. The case with no external force is quite simple because the system is symmetric with respect to $y \rightarrow-y$ and the pressure is uniform. If the system were conservative, as a normal gas, this would be a homogeneous system at thermal equilibrium, but since the system is dissipative the temperature depends on the coordinate $y$ ( $T$ has a minimum at the symmetry axis) and the problem is much less trivial. 
Since the system is purely conductive there is no velocity field, $P_{x y}=0, Q_{x}=0$ and the other fields depend only on the coordinate $y$. The set of equations (11-15) becomes

$$
\begin{array}{rlrl}
P_{x x} & =\frac{B-A}{B} p, & P_{y y} & =\frac{B+A}{B} p, \\
Q_{y}^{\prime}=-4 A p^{2} \sigma \sqrt{\frac{\pi}{m T(y)}}, & T^{\prime}=-\frac{\sigma B C}{3 A+2 B} \sqrt{\frac{\pi m}{T}} Q_{y}
\end{array}
$$

where the prime indicates derivative with respect to $y$. Notice that because there is inelasticity the pressure tensor is anisotropic in the sense that $P_{x x} \neq P_{y y}$. In fact $P_{y y}-P_{x x} \sim A \sim q$ and they do not depend on the coordinate $y$.

Next we are going to compare the implications of these equations with our molecular dynamics results. To this end we should, in principle, solve these hydrodynamic equations using the boundary conditions associated to the particular simulations that we have studied. This is not a straightforward task because, as we have mentioned at the end of the introduction, the moment expansion method (behind the previous hydrodynamic equations) does not give a good description near boundaries. For example, if we impose that a wall behaves as a stochastic wall at temperature $T_{0}$, the observed field $T$ is not expected to take that value near the wall. Later on it will be shown how we tackle this problem.

From Eq. (18) it is direct to derive that the temperature field satisfies the equation

$$
T T^{\prime \prime}+\frac{1}{2} T^{\prime 2}=k^{2} \sigma^{2} p^{2}
$$

where

$$
k^{2} \equiv \frac{4 \pi A B C}{3 A+2 B} .
$$

Since the pressure is uniform, and because of Eq. (2), this equation can also be used as an equation for the inverse of the density.

Before proceeding to solve the equations we adimensionalize the problem defining a coordinate $\xi=y / L_{y},\left(-\frac{1}{2} \leq\right.$ $\left.\xi \leq \frac{1}{2}\right)$ and we also define

$$
\begin{array}{rlrl}
\bar{n} & =\frac{N}{L_{x} L_{y}}, & n(y) & =\bar{n} n^{*}(\xi), \\
K & =\frac{k}{2 \sqrt{2}}\left(\frac{N \sigma}{L_{x}}\right), Q_{y}(y) & =\bar{n} T_{0} \sqrt{\frac{T_{0}}{m}} Q_{y}^{*}(\xi), \\
T(y) & =T_{0} T^{*}(\xi), & p & =\bar{n} T_{0} p^{*} .
\end{array}
$$

Once Eq. (19) is solved and converted to a solution for the dimensionless number density $n^{*}(\xi)$ the result is

$$
4 K|\xi|=\frac{1}{n^{*}(\xi)} \sqrt{\frac{n_{\max }^{*}-n^{*}(\xi)}{n_{\max }^{*}}}+\frac{1}{n_{\max }^{*}} \operatorname{arctanh} \sqrt{\frac{n_{\max }^{*}-n^{*}(\xi)}{n_{\max }^{*}}},
$$

where $n_{\max }^{*}$ is the value taken by $n^{*}(\xi)$ in the middle of the system, $\xi=0$. In what follows it is shown that $n_{\max }^{*}$ is a simple function of the parameter $K$ defined above. The effect of the boundaries is traded in favor of the observed values of $n_{\max }^{*}$.

The integral condition $\int n d x d y=N$ can be cast as

$$
\int_{0}^{1 / 2} n^{*} d \xi=\frac{1}{2}
$$

but since $d \xi=\left(d \xi / d n^{*}\right) d n^{*}$ the integral condition yields,

$$
\frac{n_{b}^{*}}{n_{\max }^{*}}=1-\tanh ^{2} K
$$

where $n_{b}^{*}$ is the value that $n^{*}(\xi)$ takes at the two boundaries, $n_{b}^{*}=n^{*}\left( \pm \frac{1}{2}\right)$. 
Equation (22) evaluated at $\xi=\frac{1}{2}$ yields a different condition over $n_{b}^{*}$ and it follows that

$$
n_{\max }^{*}=\frac{1}{2}+\frac{\sinh (2 K)}{4 K}
$$

This is a strong result, it says that the dimensionless number density at the center, $n_{\max }^{*}$, is determined by $K^{2}$ alone,

$$
K^{2}=a_{0}^{2} \frac{2 A B C}{3 A+2 B}
$$

where $a_{0}^{2}=N \rho_{A} / \alpha, \alpha=L_{x} / L_{y}$ is the aspect ratio of the box, and $\rho_{A}=(\pi / 4)\left(N \sigma^{2} /\left(L_{x} L_{y}\right)\right)$ is the fraction of area occupied by the disks. Since $A$ vanishes in the elastic limit and for small inelasticity coefficient $q, A \approx q$ while $B \approx 1$ and $C \approx 1$ then

$$
K^{2} \approx a_{0}^{2} q \approx \frac{1}{\alpha} q N \rho_{A} .
$$

In the quasielastic limit then, the control parameter is basically $q N \rho_{A}$. This result, for fixed area density, resembles what we obtained in the one dimensional case [18], namely, that the relevant control parameter of the one dimensional equations is $q N$.

The temperature is $T^{*}(\xi)=p^{*} / n^{*}(\xi)$ but since the pressure is uniform one may be tempted to use $p^{*}=n_{b}^{*} T_{b}^{*}$ with the value for $n_{b}^{*}$ already derived from the theory and the value $T_{b}^{*}$ imposed in the simulation. This would give a bad fit however because, as we have been emphasizing, the formalism is not reliable near the boundaries. Therefore we choose for $p^{*}$ the value $p^{*}=T_{\min }^{*} n_{\mathrm{max}}^{*}$. We know $n_{\max }^{*}$ from Eq. (25), and we take the value for $T_{\min }^{*}$ directly from our simulations. It may be said that $T_{\min }^{*}$ is a parameter to adjust our results.

The dimensionless heat flux becomes,

$$
Q_{y}^{*}(\xi)=\frac{3 A+2 B}{2 B C} \frac{1}{a_{0}} \sqrt{\frac{p^{*}}{n^{*}(\xi)}} \frac{p^{*}}{n^{*}(\xi)^{2}} \frac{d n^{*}}{d \xi} .
$$

\section{SIMULATION-THEORY COMPARISON}

In this section we compare the simulational results with the values given by three formalisms which use as reference function: $(i) f_{\mathrm{M}}$, (ii) $f_{\mathrm{HCS}}$ and $($ iii $) f_{0}$. We are calling $f_{\mathrm{HCS}}$ the function like $f_{0}$ but with $\kappa$ defined with the values given in Eq. (9).

Since the formalisms differ by terms of higher order in $q$ their predictions are quite similar unless $q N$ is large enough. Typically the Maxwellian theory and the one based on $f_{\text {HCS }}$ are valid until about $q N=20$ and are reasonable until $q N=40$ while the theory based on $f_{0}$, with an adjusted $\kappa$, is valid for values of $q N$ up to 200, and reasonably good until about $q N \approx 300$.

\section{A. Simulational setup}

We have performed simulations of a two dimensional system of $N=2300, N=3600, N=10000$, and $N=19600$ inelastic hard disks inside a $L \times L$ box with lateral periodic boundary conditions while the upper and lower walls are kept at granular temperature $T_{0}=1$. The area fraction covered by the disks was chosen to be $\rho_{A}=0.01$ (in which case the nonideal corrections to the equation of state are less than $2 \%$ ) while the $q N$ dissipation parameter ranges from $q N=10$ up to $q N=400$. In the $N=2300$ case, the smallest simulated system, the ratio between the mean free path and the linear size of the system (Knudsen number) is 0.065 and it is smaller in the other cases. This value guarantees that not too close to the walls the fluid has a hydrodynamic behavior. The wall temperature $T_{0}$ is imposed sorting the velocity of the bouncing particles as if they were coming from a heat bath at $T=T_{0}$.

In every simulation the system was relaxed from an initial condition for a sufficiently long time. After the relaxation we measure local time averages of the main moments of the distribution (i.e., $n, \vec{v}, T, p_{i j}, \vec{Q}$ ) inside each one of a set of square cells. Taking advantage of the translation invariance in the $X$ direction, it is natural to take horizontal averages getting, in this way, smooth vertical profiles for the observed hydrodynamic fields. 


\section{B. Theory and simulation comparison}

In order to compare theory and simulation some analysis is needed because size effects are quite noticeable unless the number $N$ of particles is above about 3600. We use expression (25) for $n_{\max }^{*}$ as our point of contact between theory and simulation and proceed to adjust the coefficients $b_{k}$ in Eq. (8) so that $K$ takes the values that make theory give the observed values for $n_{\text {max }}^{*}$.

With this aim we first write $K$ as a rational expression $K=a_{0} \sqrt{q}\left(1+a_{1} q\right) /\left(1+a_{2} q\right)$ and find the values of the $a_{k}$ so that Eq. (25) reproduces the observed values of $n_{\max }^{*}$. We have to adjust $a_{0}$ in spite of its definition, given under Eq. (26), because the effective values for the number of particles, the global density and the aspect ratio get distorted since there is a layer near the thermalizing walls which does not behave hydrodynamically. Once this expression for $K$ is fixed, we invert (26) to obtain values for $\kappa$ as a function of $q$. The size effect is in $a_{0}$ alone and $\kappa(q)$ is approximately the same for systems with $N$ larger than about 3600, as shown in Fig. 11. In this figure there is a solid line which is Eq.(8) with

$$
b_{1}=-22.541, \quad b_{2}=6.19187, \quad b_{3}=79.0362
$$

The discrepancies between this curve and the empirical values of $\kappa$ are less than $2 \%$ for the whole range of $q$ considered. More in detail, Fig. 11 shows the behavior of $\kappa(q)$ for systems of different size. It is seen that in the case of $N=2300$ (solid circles) $\kappa(q)$ is dependent on the system's size, while the predictions in the cases $N=3600,10000$, and 19600 differ among themselves by less than $2 \%$. Only the smallest simulated system $(N=2300)$ departs from this otherwise universal shape.

In the following we present the results corresponding to the $N=10000$ case.

a. The density: As a first step and in order to check the validity of the kinetic description (no clustering, [5]) we have plotted the final configurational positions of the particles (not shown here) for different values of $q N$ up to $q N=400$, founding that clustering begins at about $q N \approx 300$. In fact, we have detected that the number of collisions per unit time increases dramatically shortly after $q N=300$.

As a second step we check the validity of Eq. (25) (which for fixed geometry depends only on $q N$ ) with simulations. The top Fig. 2 shows both $n_{\max }^{*}$ (growing curves with $q N$ ) and $n_{b}^{*}$ (the decreasing curves). It is seen how well the values of $n_{\max }^{*}$ (solid circles) come from the $f_{0}$ distribution (there should be no surprise as these are the fitted data), compared with the prediction using the other two distributions. This graph shows that $n_{\max }^{*}$ with the other two formalisms is good only up to $q N \approx 40$.

Also at top Fig. 2 shows the values predicted by Eq. (24) and the observed values of $n_{b}^{*}$ (see caption), and it is seen that all theoretical schemes give values close to the simulational results in the considered range of $q N$. This is the only case where, for large $q N$, predictions coming from $f_{\mathrm{M}}$ are better that those coming from $f_{0}$, but we do not believe there is anything deep here since our moment expansion method is not reliable near walls.

Figure 2, bottom, compares theory and simulational density profiles for $q N=30$ and $q N=200$. As it has already been explained, the value $n_{\max }^{*}$ in Eq. (22) is fixed by Eq. (25) and there are no extra parameters to adjust. It is seen that theory in all cases $\left(f_{\mathrm{M}}, f_{\mathrm{HCS}}\right.$ and $\left.f_{0}\right)$ give good agreement for low values of $q N$. However predictions for $q N=200$, when using $f_{\mathrm{M}}$ or $f_{\mathrm{HCS}}$ fail. It is amazing how large $q N$ can be when $f_{0}$ is used. In the last case the parameter $K$ is adjusted only from the knowledge of $n_{\max }^{*}$ and it accurately predicts the behavior in almost all the volume.

From the figure it can be appreciated that near the walls $(\xi= \pm 0.5)$, as mentioned in the introduction, the theory does not predict well the behavior of $n^{*}(\xi)$.

$b$. The temperature: It has already been mentioned that the temperature profiles exhibit a minimum at the center and there is a temperature jump at the boundaries. In Fig. 3 we show the simulational results for $T_{\min }^{*}=T^{*}(\xi=0)$ and temperature $T_{b}^{*}=T^{*}(\xi= \pm 1 / 2)$. It is seen that for $q N=40$ the temperature of the fluid by the walls is about $40 \%$ lower than the imposed value. This effect is due to dissipation and in $1 \mathrm{D}$ it has been shown to be a $\mathcal{O}(q N)$ effect 18 .

Because at present we have no theory to describe the temperature jump that takes place near the thermal walls, and we know that Grad's method does not give good results near boundaries, we have chosen the observed value of the temperature at mid height, $T_{\min }^{*}$, as the value to use in the formalism. The observed values decrease with $q N$ and, in the case $N=10000$, we have adjusted them with the following expression

$$
\begin{aligned}
\ln \left(T_{\min }\right)= & -0.0163259 q N+5.2765610^{-5}(q N)^{2} \\
& -1.8639610^{-7}(q N)^{3}+4.24110^{-10}(q N)^{4} \\
& -4.2387810^{-13}(q N)^{5}
\end{aligned}
$$

whose faithfulness is shown in Fig. 3. 
Since the pressure is uniform the temperature profile can be written directly as

$$
T^{*}(\xi)=\frac{T_{\min }^{*} n_{\max }^{*}}{n^{*}(\xi)} .
$$

Figure 1 shows the simulational and theoretical temperature profiles for some values of $q N$. The three upper curves show the profiles for small $q N$ values $(q N=10,20,30)$ and it is seen that the predictions using $f_{0}$ (solid line) give an excellent fit to the simulational data while for the results obtained using $f_{\mathrm{M}}$ or $f_{\mathrm{HCS}}$ (dashed and light lines respectively) the fit is only fair. The lowest curves shows $T$ profiles for larger values of $q N(q N=50,100,200)$. In this case only the formalism based on $f_{0}$ give an acceptable description and the agreement is very good up to $q N=200$. In the case of $q N=275$, not shown, there is still a reasonable agreement when $f_{0}$ is used.

c. The pressure: Now that the values of $n_{\max }^{*}$ and $T_{\min }^{*}$ are known and since the pressure is uniform (both in theory and simulationally) and theory asserts that $p^{*}=T_{\min }^{*} n_{\max }^{*}$, then we have the value of $p^{*}$.

From the value for the pressure and the set of equations (18) one gets the theoretical values for $P_{y y}$ in terms of the pressure tensor. These values are compared with our simulational data in Fig. F. Again the comparison is good when the formalism with $f_{0}$ is used and it is only fairly good with the other two formalisms.

As it has already been mentioned after Eq. (18), the two diagonal terms of the pressure tensor are not equal because the system is anisotropic.

d. The heat current: Equation (28) gives the theoretical expression for the heat current $Q_{y}^{*}$. At top Fig. 6 shows simulational data and theoretical predictions for small values of $q N$. The three formalism predict well the observed values.

At bottom in Fig. 6 it is possible to see that for $q N=200$ only the formalism using $f_{0}$ fits the observed data, while the other two fail badly. In the case of $q N=275$ the agreement is still reasonable within about $3 \%$.

\section{FINAL COMMENTS}

In this article we have studied a bidimensional granular system of $N$ inelastic hard disks (normal restitution coefficient $r$ ). The system is placed in a rectangular box and it is kept in a stationary regime with upper and lower walls at granular temperature $T_{0}$. The lateral walls are periodic. The quantity $q N$ has been used as control parameter, where $q=\frac{1-r}{2}$, and simulations were made with different values of $N$ ranging from $N=2300$ to 19600 . If the system were conservative it would remain in a perfectly homogeneous state at temperature $T_{0}$ with a homogeneous area density $\rho_{A}=0.01$. Because there is dissipation the dimensionless number density $n^{*}$ has a maximum in the middle, $n_{\max }^{*}$, and the dimensionless temperature $T^{*}$ has a minimum, $T_{\min }^{*}$, also at the center of the system. The pressure is uniform and $p^{*}=n_{\max }^{*} T_{\min }^{*}$.

Hydrodynamic equations were derived using a moment expansion method. This method has the fourth cumulant, $\kappa$, of the velocity distribution as a parameter and three possible $\kappa$ 's where considered. These are: $\kappa=0$ which implies that the reference distribution function in a Maxwellian, and two $\kappa$ 's of the form (8), one with parameters $b_{k}$ as in (9) which corresponds to using $f_{\mathrm{HCS}}$ as the reference function and finally using the values $b_{k}$, Eq. (29), numerically determined to ensure that the correct values of the density at the middle of the system come out. The last case gives our reference function $f_{0}$.

The empirical rational form of $\kappa=\kappa(q)$, Eq. (8), in the case of the successful distribution $f_{0}$ turns out to be independent of the size of the system.

When this $f_{0}$ is used, the comparison between the predictions and simulation results for $n_{\max }^{*}, p^{*}, P_{x x}^{*}, P_{y y}^{*}$ against dissipation, and the density, temperature and heat flux profiles are very good.

The obvious conclusion is that the formalism based on $f_{0}$ gives an excellent description of the behavior of the system for values of $q N$ up to 200 and it gives a reasonable description up to $q N$ nearly 300 (slightly above $q N \sim 300$ clustering begins), while the other formalisms fail beyond about $q N=40$.

Even though the fourth cumulant $\kappa$ of $f_{0}$ is obtained to fit the results of a particular hydrodynamic regime, we expect that the theory with this expression for $\kappa$ is still valid for other regimes.

\section{ACKNOWLEDGMENTS}

This work has been partly financed by Fondecyt research grant 296-0021 (R.R.), Fondecyt research grant 1990148 (D.R.), Fondecyt research grant 197-0786 (P.C.) and by FONDAP grant 11980002. One of us (R.S.) acknowledges a grant from MIDEPLAN. 
[1] H.M. Jaeger and S.R. Nagel, Science 2551523 (1992); H.M. Jaeger, S.R. Nagel, and R.P. Behringer, Phys. Today 49 , 32 (1996); H.M. Jaeger, S.R. Nagel, and R.P. Behringer, Rev. Mod. Phys. 681259 (1996).

[2] J.A.C. Gallas, H.J. Herrmann and S. Sokolowski. Physica A 189, 437 (1992); Y. Zhang and C.S. Campbell. J. Fluid Mech. 237, 541 (1992); S. Luding, H.J. Herrmann and A. Blumen. Phys. Rev. E 503100 (1994); S. Warr, J.M. Huntley and G.T.H. Jacques. Phys. Rev. E 52, 5583 (1995); N. Mujica and F. Melo, Phys. Rev. Lett. 80 5121-5124 (1998).

[3] C.S. Campbell, Annu. Rev. Fluid Mech. 22, 57 (1990).

[4] J.T. Jenkins and S.B. Savage, J. Fluid Mech. 130, 187 (1983); C. Lun, S. Savage, D. Jeffrey and R.P. Chepurnuy, J. Fluid Mech. 140, 223 (1984); J.T. Jenkins and M.W. Richman, Arch. Rat. Mech. Anal. 87, 355 (1985); J.T. Jenkins and M.W. Richman, Phys. Fluids 28, 3485 (1985); P.K. Haff, J. Fluid Mech. 134, 401 (1983).

[5] I. Goldhirsch and G. Zanetti, Phys. Rev. Lett. 70, 1619 (1993).

[6] S. McNamara, Phys. Fluids A 5, 3056 (1993).

[7] A. Goldstein and M. Shapiro, J. Fluid Mech 282, 75 (1995).

[8] S. McNamara and W.R. Young, Phys. Rev. E 53, 5089 (1996); R. Brito and M.H. Ernst , Europhys. Lett. 43, 497 (1998); G. Peng and T. Ohta, Phys. Rev. E 58, 4737 (1998); T.P.C. van Noije, M. H. Ernst, and R. Brito, Phys. Rev. E 57, R4891 (1998).

[9] J.J. Brey, F. Moreno, and J.W. Dufty Phys. Rev. E 54, 445 (1996); J.J. Brey, M.J. Ruiz-Montero and D. Cubero, Phys. Rev. E 54, 3664 (1996).

[10] R. Soto, M. Mareschal and D. Risso, Phys. Rev. Letter, 83, 5003 (1999).

[11] T.P.C. van Noije, M.H. Ernst, Granular Matter, 1, 57 (1998).

[12] N.V. Brilliantov and T. Pöschell, Phys. Rev. E to appear in Phys. Rev. E 61, (1999).

[13] J.H. Ferziger and H.G. Kaper Mathematical Theory of Transport Processes in Gases, North-Holland 1972.

[14] H. Grad, Principles of the Kinetic Theory of Gases in Handbuch der Physik, Vol. XII, edited by S. Flüge, Springer, (1958); Theory of Rarefied Gases in Rarefied Gas Dynamics, edited by F. Devienne, Pergamon Press, 1960.

[15] J.J. Brey, J.W. Dufty, C.S. Kim, and A. Santos. Phys. Rev. E 58, 4638 (1998); V. Garzó and J.W. Dufty, Phys. Rev. E 59, 5895 (1999).

[16] D. Risso and P. Cordero, Phys. Rev. E 56, 489-496 (1997). Erratum: Phys. Rev. E 57, 7365 (1998).

[17] H. Struchtrup, W. Weiss, Phys. Rev. Letters 80, 5048 (1998).

[18] Rosa Ramírez and P. Cordero, Phys. Rev. E 59 656-664 (1999).

[19] J.J. Brey and B. Cubero, Phys. Rev. E 57, 2019 (1998). 
FIG. 1. The fourth cumulant $\kappa$ against $q$ for systems of different size. The empirical values of $\kappa$ are represented by triangles for $N=19600$, empty circles for $N=10000$, empty squares for $N=3600$, and solid circles for $N=2300$. The solid line corresponds to our empirical fit. See text.

FIG. 2. At top the predicted and observed values for the density $n_{\max }^{*}$ at the center of the box $(\xi=0)$ and $n_{b}^{*}$ near the boundaries $(\xi= \pm 0.5)$. The solid (open) circles correspond to the simulational values for $n_{\max }^{*}\left(n_{b}^{*}\right)$, the light-dashed (heavy-dashed) line corresponds to the theoretical prediction using $f_{\mathrm{HCS}}\left(f_{\mathrm{M}}\right)$. The solid line corresponds to our empirical adjustment (see text). At bottom the predicted and observed density profiles for two different values of $q N$. The open (solid) circles correspond to $q N=30(q N=200)$. The light-dashed (heavy-dashed) line corresponds to the theoretical prediction using $f_{\mathrm{HCS}}\left(f_{\mathrm{M}}\right)$. The solid line corresponds to the prediction stemming from $f_{0}$.

FIG. 3. Temperature $T_{\min }^{*}$ at the center of the channel (open circles) against dissipation $q N$. The solid line corresponds to a fit using Eq. (30). The solid circles are the observed values for the temperature $T_{b}^{*}$ near the boundaries

FIG. 4. From top to bottom temperature profiles corresponding to $q N=5,10,30,50,100$ and 200 . The empty circles are the simulational results, the dashed line (light solid line) correspond to the predictions obtained with $f_{\mathrm{M}}\left(f_{\mathrm{HCS}}\right)$. The heavy solid lines are the theoretical results when $f_{0}$ is used.

FIG. 5. Simulational and theoretical values for the components of the pressure tensor against dissipation $q N$. At top (bottom) the $P_{x x}\left(P_{y y}\right)$ component. In light-solid line (dashed line) the theoretical results when using $f_{\mathrm{HCS}}$ distribution $\left(f_{\mathrm{M}}\right.$ distribution). The heavy-solid line is the theoretical result when using the $f_{0}$ distribution.

FIG. 6. Simulational and theoretical values for the heat flux profiles. At top the three curves and set of simulational data correspond to $q N=5$ (circles), $q N=20$ (squares) and $q N=30$ (rhombus). The dashed and light-solid lines correspond to the predictions using $f_{\mathrm{M}}$ and $f_{\mathrm{HCS}}$ respectively, the heavy solid line corresponds to $f_{0}$. At bottom are the observed values of $Q_{y}^{*}$ when $q N=200$ and, the theoretical predictions when using $f_{0}$ (heavy solid line), $f_{\mathrm{M}}$ (dashed line) and $f_{\mathrm{HCS}}$ (light-solid line). 